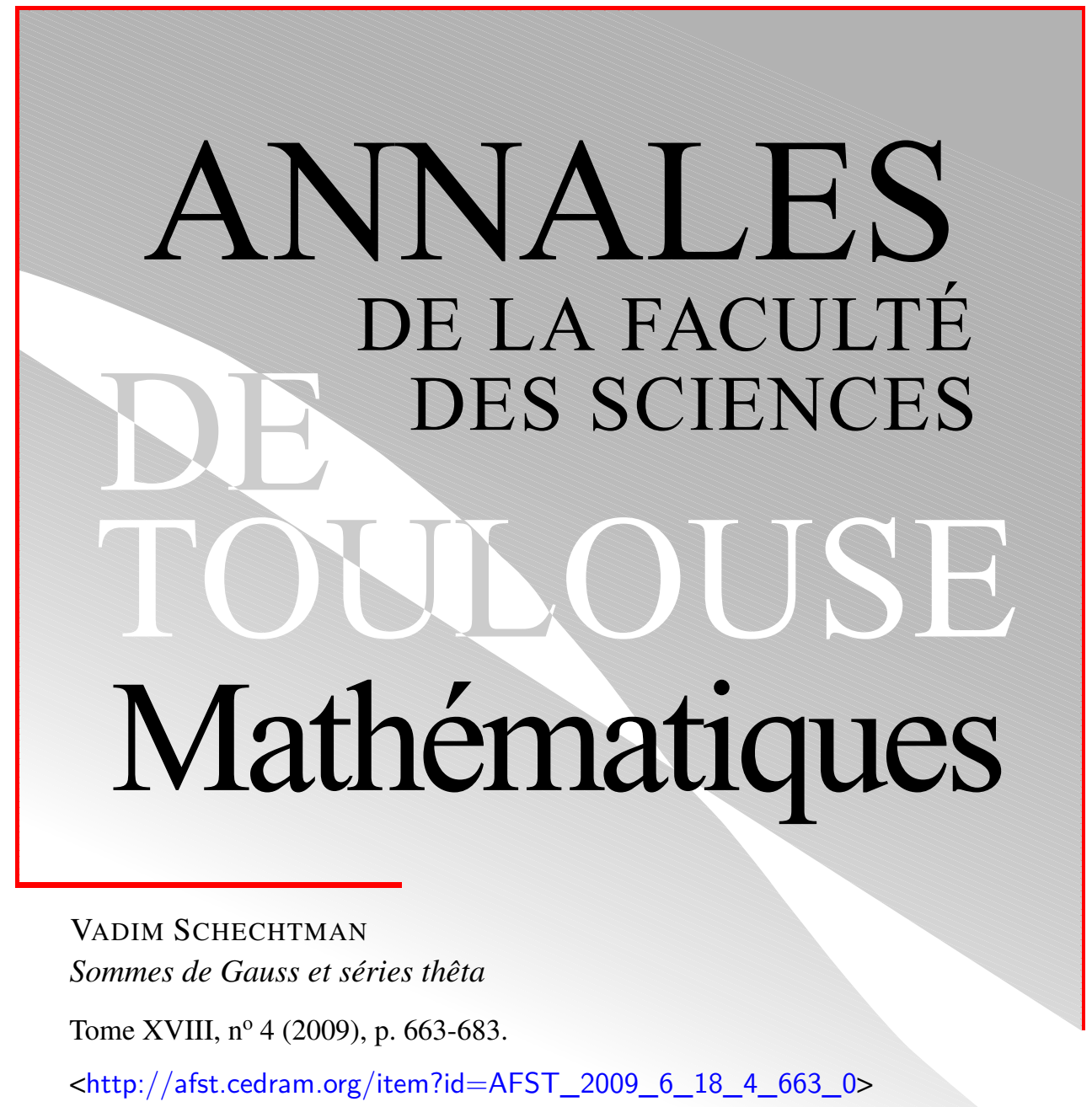

(C) Université Paul Sabatier, Toulouse, 2009, tous droits réservés.

L'accès aux articles de la revue "Annales de la faculté des sciences de Toulouse Mathématiques » (http://afst.cedram.org/), implique l'accord avec les conditions générales d'utilisation (http://afst.cedram.org/ legal/). Toute reproduction en tout ou partie cet article sous quelque forme que ce soit pour tout usage autre que l'utilisation à fin strictement personnelle du copiste est constitutive d'une infraction pénale. Toute copie ou impression de ce fichier doit contenir la présente mention de copyright.

\title{
cedram
}

Article mis en ligne dans le cadre du

Centre de diffusion des revues académiques de mathématiques

http://www.cedram.org/ 


\section{Sommes de Gauss et séries thêta}

(Summatio quarumdam serierum singularium)

VADIM SCHECHTMAN ${ }^{(1)}$

\section{Table des matières}

Introduction . . . . . . . . . . . . . .663

1 L'intégrale de Kronecker . . . . . . . . . . . . . . . . 664

2 Réciprocité quadratique . . . . . . . . . . . 669

3 La formule sommatoire de Poisson . . . . . . . . 672

4 Une valeur limite d'une série thêta . . . . . . . . .674

$5 \quad$ La formule de produit de Gauss . . . . . . . . . . 677

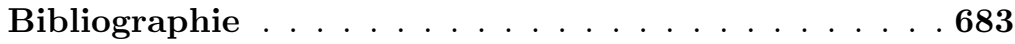

\section{Introduction}

Comme l'a dit Hecke, $[\mathrm{H}], \S 55$, "die genauere Kenntnis des Verhaltens einer analytischen Funktion in der Nähe ihrer singulären Stellen eine Quelle von arithmetischen Sätzen ist", "la connaissance plus précise du comportement d'une fonction analytique au voisinage de ses singularités est une source de théorèmes arithmétiques".

Les résultats classiques de Gauss, Cauchy et Kronecker, qu'on va présenter dans cette note, peuvent servir comme un bon exemple de ce principe. Cauchy est peut-être le premier à avoir remarqué (cf. [C, (a)]) que la valeur limite de la série

$$
\theta(t)=\sum_{n=-\infty}^{\infty} e^{-\pi n^{2} t}
$$

(*) Reçu le 20/01/08, accepté le 17/04/09

(1) Institut de Mathématiques de Toulouse, Université Paul Sabatier, 31062 Toulouse CEDEX 9 
au bord de son domaine de convergence $\Re(t)>0$, en un point $t=i r$, avec $r \in Q$ (i.e. quand $q=e^{-\pi t}$ est une racine de l'unité), est égale à ce qu'on appelle aujourd'hui la somme de Gauss (quadratique). La propriété fondamentale de $\theta(t)$ est sa modularité:

$$
\theta\left(t^{-1}\right)=t^{1 / 2} \theta(t)
$$

Kronecker (cf. [K] (a)) a démontré que (M) entraîne (en fait, Kronecker affirme que (M) est équivalente à) la formule de réciprocité pour les sommes de Gauss (cf. 1.7 et 4.4 ci-dessous), qui à son tour est équivalent à la loi de réciprocité quadratique (cf. (2.5) ci-dessous).

Comment prouve-t'-on la formule (M)? Il y a plusieurs voies ; Cauchy a découvert qu'on peut démontrer ( $\mathrm{M}$ ) (ainsi que sa généralisation, la formule sommatoire de Poisson (plutôt de Cauchy, comme dit André Weil)) en appliquant la formule des résidus, cf. $\S 3$ ci-dessous.

On peut agir directement. Dans $\S 1$ on prouve, en suivant Siegel [S] et Chandrasekharan $[\mathrm{Ch}]$, la formule de réciprocité pour les sommes de Gauss, en employant la formule de résidus à l'intégrale de Kronecker. Dans §2, on en déduit la loi de réciprocité quadratique, cf. [K (a)], [Ch].

Dans $\S 4$ on obtient, avec Kronecker, les sommes de Gauss comme valeurs limites de $\theta(t)$ et on en déduit la formule de réciprocité.

Le dernier chapitre $\S 5$ est de nature plutôt algébrique. Ici on déduit avec Gauss [G] un développement des sommes de Gauss en produit ; et de là le signe de cette somme. En passant à la limite, on obtient une développement d'une fonction thêta en produit infini ${ }^{1}$.

Ces notes ont fait l'objet de deux exposés à Toulouse en mai 2005. Je suis reconnaissant au rapporteur anonyme pour ses remarques utiles.

\section{L'intégrale de Kronecker}

Cf. [S] et [Ch, Ch. V].

1.1. Considérons l'intégrale

$$
f(X)=f(X, \tau)=\int_{C} \Phi(u) d u
$$

(1) On remarque que les identités utilisés par Gauss au cours des calculs peuvent être considérées comme les predecesseurs de célèbres identités de Rogers-Ramanujan 
où

$$
\Phi(u)=\Phi(u, X)=\Phi(u, X, \tau)=\frac{e^{\pi i \tau u^{2}+2 \pi i X u}}{e^{2 \pi i u}-1},
$$

$u, X, \tau \in C, \operatorname{Re} \tau>0, C$ étant la droite $\frac{1}{2}+r e^{\pi i / 4}, \infty<r<\infty$.

Montrons que l'intégrale converge. Plus généralement, considérons une droite

$$
C_{c}: u=c+r e^{\pi i / 4}, c \in R,-\infty<r<\infty
$$

On a

$$
\left|e^{\pi i \tau u^{2}+2 \pi i X u}\right|=e^{-\pi \operatorname{Im}\left(\tau u^{2}+2 X u\right)}
$$

or

$$
\tau u^{2}+2 X u=i \tau r^{2}+2 e^{\pi i / 4}(\tau c+X) r+(\tau c+2 X) c
$$

d'où

$$
\operatorname{Im}\left(\tau u^{2}+2 X u\right) \geqslant \operatorname{Re}(\tau) r^{2}-\left|2 e^{\pi i / 4}(\tau c+X)\right||r|-|(\tau c+2 X) c|
$$

Donc

$$
\left|e^{\pi i \tau u^{2}+2 \pi i X u}\right| \leqslant A e^{-\pi r^{2} \operatorname{Re} \tau+B|r|}
$$

D'autre part,

$$
\left|e^{2 \pi i u}-1\right| \geqslant|1-| e^{2 \pi i u}||=\left|1-e^{-\sqrt{2} \pi r}\right| \geqslant \frac{1}{2}
$$

pour $|r|$ (ou $|u|)$ assez grand. Il s'en suit que

$$
|\Phi(u)| \leqslant A^{\prime} e^{-\pi r^{2} \operatorname{Re} \tau+B|r|}
$$

pour $|r|$ assez grand; d'où la convergence.

1.2. Considérons le contour parallélogramme $\gamma_{A}$ dans le plan complexe $C=\{z=x+i y\}$, formé par les segments $L_{+}$et $L_{-}$des droites $y=A$ et $y=-A$ situés entre les droites $C=C_{1 / 2}$ et $C_{n+1 / 2}$, où $A>0, n \in Z, n>0$, et les segments de ces droites entre $L_{ \pm}$; ce contour est orienté positivement.

Par le théorème de Cauchy

$$
\int_{\gamma_{A}} \Phi(u) d u=\sum_{k=1}^{n} e^{\pi i \tau k^{2}+2 \pi i X k}
$$

car $\Phi(u)$ n'a de poles qu'au points réels $k=1, \ldots, n$, avec les résidus correspondants. 
L'estimation (1.1.3) implique que

$$
\lim _{A \rightarrow \infty} \int_{L_{ \pm}} \Phi(u) d u=0
$$

d'où

$$
\left\{\int_{C_{n+1 / 2}}-\int_{C}\right\} \Phi(u) d u=\sum_{k=1}^{n} e^{\pi i \tau k^{2}+2 \pi i X k}=: g(\tau, X ; n)
$$

Par contre,

$$
\Phi(u+n)=e^{\pi i \tau n^{2}+2 \pi i X n} \Phi(u, X+\tau n),
$$

donc

$$
\int_{C_{n+1 / 2}} \Phi(u) d u=e^{\pi i \tau n^{2}+2 \pi i X n} f(X+\tau n),
$$

d'où l'équation

$$
e^{\pi i \tau n^{2}+2 \pi i X n} f(X+\tau n)-f(X)=g(\tau, X ; n)
$$

1.3. On a

$$
\begin{gathered}
f(X+1)-f(X)=\int_{C} \frac{e^{\pi i \tau u^{2}}}{e^{2 \pi i u}-1}\left\{e^{2 \pi i(X+1) u}-e^{2 \pi i X u}\right\} d u \\
=\int_{C} e^{\pi i \tau u^{2}+2 \pi i X u} d u=e^{-\pi i X^{2} / \tau} \int_{C} e^{\pi i \tau(u+X / \tau)^{2}} d u=e^{-\pi i X^{2} / \tau} \int_{C_{1 / 2+X / \tau}} e^{\pi i \tau u^{2}} d u \\
=e^{-\pi i X^{2} / \tau} \int_{C_{0}} e^{\pi i \tau u^{2}} d u
\end{gathered}
$$

(par le théorème de Cauchy). Or $C_{0}$ est donnée par l'équation $u=r e^{\pi i / 4}$, donc

$$
\int_{C_{0}} e^{\pi i \tau u^{2}} d u=e^{\pi i / 4} \int_{-\infty}^{\infty} e^{-\pi \tau r^{2}} d r=: e^{\pi i / 4} I_{\tau}
$$

On en conclut que

$$
f(X+1)-f(X)=e^{\pi i\left(1 / 4-X^{2} / \tau\right)} I_{\tau}
$$

1.4. Il s'en suit que $(m \in Z, m>0)$ :

$$
f(X+m)-f(X)=I_{\tau} \cdot \sum_{\nu=0}^{m-1} e^{\pi i\left[1 / 4-(X+\nu)^{2} / \tau\right]}
$$


Remplaçons ici $X$ par $X+\tau n-m$ :

$$
\begin{gathered}
f(X+\tau n)-f(X+\tau n-m)=I_{\tau} \cdot \sum_{\nu=0}^{m-1} e^{\pi i\left[1 / 4-(X+\tau n-m+\nu)^{2} / \tau\right]}= \\
=I_{\tau} \cdot \sum_{\nu=1}^{m} e^{\pi i\left[1 / 4-(X+\tau n-\nu)^{2} / \tau\right]}
\end{gathered}
$$

Donc, en tenant compte de (1.2.1) :

$$
\begin{gathered}
e^{\pi i \tau n^{2}+2 \pi i X n} f(X+\tau n-m)-f(X)= \\
=g(\tau, X ; n)-I_{\tau} e^{\pi i \tau n^{2}+2 \pi i X n} \sum_{\nu=1}^{m} e^{\pi i\left[1 / 4-(X+\tau n-\nu)^{2} / \tau\right]}= \\
=g(\tau, X ; n)-I_{\tau} \sum_{\nu=1}^{m} e^{\pi i\left(1 / 4-(X-\nu)^{2} / \tau\right.}= \\
=g(\tau, X ; n)-I_{\tau} e^{\pi i\left(1 / 4-X^{2} / \tau\right)} g(-1 / \tau, X / \tau ; m)
\end{gathered}
$$

1.5. On définit pour $m, n \in Z$

$$
g(m, n):=g(m / n, m / 2 ; n)=\sum_{k=1}^{|n|} e^{\pi i\left(m k^{2} / n+m k\right)}
$$

Posons dans (1.4.2) $\tau=m / n, X=m / 2, m, n>0$ :

$$
g(m, n)=I_{m / n} e^{\pi i(1-m n) / 4} g(-n / m, n / 2 ; m)=I_{m / n} e^{\pi i(1-m n) / 4} g(-n, m)
$$

Remarquons que $g(1,1)=g(-1,1)=1$, d'où

$$
I_{1}=\int_{-\infty}^{\infty} e^{-\pi r^{2}} d r=1
$$

il s'en suit que

$$
I_{\tau}=\int_{-\infty}^{\infty} e^{-\pi \tau r^{2}} d r=\frac{1}{\sqrt{\tau}}(\tau>0)
$$

Donc

$$
g(m, n)=\sqrt{n / m} e^{\pi i(1-m n) / 4} g(-n, m) \quad(m, n>0)
$$

1.6. Calcul d'intégrale de Poisson. Considérons la fonction

$$
\begin{aligned}
\Psi(u)=\frac{e^{\pi i u^{2}+\pi i u}}{e^{2 \pi i u}-1} & =\frac{e^{\pi i u^{2}}}{e^{\pi i u}-e^{-\pi i u}}=\frac{e^{\pi i u^{2}}}{2 i \sin (\pi u)} \\
& -667-
\end{aligned}
$$


Par le théorème de Cauchy,

$$
\left\{\int_{C_{3 / 2}}-\int_{C}\right\} \Psi(u) d u=2 \pi i \cdot \operatorname{Res}_{u=1} \Psi(u)=1
$$

D'un autre côté,

$$
\begin{aligned}
\left\{\int_{C_{3 / 2}}-\int_{C}\right\} \Psi(u) d u & =\int_{C}(\Psi(u+1)-\Psi(u)) d u=\int_{C} e^{\pi i u^{2}+\pi i u} d u= \\
=\int_{C} e^{\pi i\left[(u+1 / 2)^{2}-1 / 4\right]} d u= & e^{-\pi i / 4} \int_{C} e^{\pi i(u+1 / 2)^{2}} d u=e^{-\pi i / 4} \int_{C_{1}} e^{\pi i u^{2}} d u= \\
& =e^{-\pi i / 4} \int_{C_{0}} e^{\pi i u^{2}} d u
\end{aligned}
$$

(de nouveau par Cauchy). Or, sur $C_{0}$, on a $u=r e^{\pi i / 4}$, donc $u^{2}=r^{2} e^{\pi i / 2}=$ $i r^{2}, e^{\pi i u^{2}}=e^{-\pi r^{2}}$, d'où

$$
\int_{C_{0}} e^{\pi i u^{2}} d u=\int_{-\infty}^{\infty} e^{-\pi r^{2}} e^{\pi i / 4} d r=e^{\pi i / 4} \int_{-\infty}^{\infty} e^{-\pi r^{2}} d r
$$

Il s'en suit que

$$
\left\{\int_{C_{3 / 2}}-\int_{C}\right\} \Psi(u) d u=\int_{-\infty}^{\infty} e^{-\pi r^{2}} d r
$$

donc

$$
\int_{-\infty}^{\infty} e^{-\pi r^{2}} d r=1
$$

1.7. Théorème (loi de réciprocité pour les sommes de Gauss). Si $m, n \in$ $Z, m, n \neq 0$, alors

$$
\frac{1}{\sqrt{|n|}} g(m, n)=e^{\pi i(1-|m n|) \operatorname{sgn}(m n) / 4} \frac{1}{\sqrt{|m|}} g(-n, m),
$$

où

$$
g(m, n)=\sum_{k=1}^{|n|} e^{\pi i m k^{2} / n+\pi i m k}
$$

Pour $m, n>0$ ceci est (1.5.4). Remarquons que

$$
g(-m,-n)=g(m, n)
$$


Il s'en suit que (1.7.1) est vrai pour $(m, n)$ si et seulement si il l'est pour $(-m,-n)$.

Si $m>0$ et $n<0$, alors $-n, m>0$, donc

$$
m^{-1 / 2} g(-n, m)=(-n)^{-1 / 2} e^{\pi i(1+m n)} g(-m,-n),
$$

$\mathrm{ou}$

$$
(-n)^{-1 / 2} g(-m,-n)=e^{-\pi i(1+m n)} m^{-1 / 2} g(-n, m),
$$

ce qui est (1.7.1) en tenant compte de (1.7.3), cqfd.

\section{Calcul des sommes de Gauss}

2.1. Puisque $k^{2} \equiv k(\bmod 2)$, on peut reécrire la définition de la somme de Gauss sous une forme

$$
g(m, n)=\sum_{k=1}^{|n|} e^{\pi i k^{2} m(n+1) / n}
$$

Remarquons que

$$
g(m, 1)=g(m,-1)=1
$$

2.2. Supposons dorénavant que $n$ est un nombre premier impair et $(m, n)=1$. Alors $n+1$ est pair, $(n, n+1)=1$, et

$$
\eta:=e^{\pi i(n+1) / n}
$$

est une racine $n$-ième de l'unité, $\eta \neq 1$. On a

$$
g(m, n)=1+\sum_{k=1}^{n-1} \eta^{m k^{2}}=1+2 \sum_{\rho} \eta^{m \rho},
$$

où $\rho$ parcourt les résidus quadratiques modulo $n$.

D'un autre côté, si $\nu$ parcourt les nonrésidus quadratiques modulo $n$,

$$
1+\sum_{\rho} \eta^{m \rho}+\sum_{\nu} \eta^{m \nu}=\sum_{k=0}^{n-1} \eta^{m k}=0,
$$

d'où

$$
g(m, n)=\sum_{\rho} \eta^{m \rho}-\sum_{\nu} \eta^{m \nu}=\sum_{k=1}^{n-1}\left(\frac{k}{n}\right) \eta^{m k}
$$


Il s'en suit que

$$
g(m, n)=\left(\frac{m}{n}\right) g(1, n)
$$

2.3. ThÉORÈme. - Si $n$ est un nombre premier impair, alors

$$
g(1, n)=e^{\pi i(1-n) / 4} \sqrt{n}
$$

Donc

$$
\left(\frac{m}{n}\right)=n^{-1 / 2} e^{\pi i(n-1) / 4} g(m, n)
$$

En effet,

$$
n^{-1 / 2} g(1, n)=e^{\pi i(1-n) / 4} g(-n, 1)=e^{\pi i(1-n) / 4}
$$

2.4. THÉORÈME. -

$$
\left(\frac{-1}{n}\right)=(-1)^{(n-1) / 2}
$$

En effet,

$$
\left(\frac{-1}{n}\right)=n^{-1 / 2} e^{\pi i(n-1) / 4} g(-1, n)
$$

Or

$$
n^{-1 / 2} g(-1, n)=e^{\pi i(n-1) / 4} g(n,-1)=e^{\pi i(n-1) / 4},
$$

d'où l'assertion.

2.5. ThÉORÈME. - Soient $m, n$ des nombres premiers impairs distincts. Alors

$$
\left(\frac{m}{n}\right)=(-1)^{(m-1)(n-1) / 4}\left(\frac{n}{m}\right)
$$

En effet,

$$
\begin{gathered}
\left(\frac{m}{n}\right)=n^{-1 / 2} e^{\pi i(n-1) / 4} g(m, n)= \\
=e^{\pi i(n-1) / 4} e^{\pi i(1-m n) / 4} m^{-1 / 2} g(-n, m)= \\
=e^{\pi i(n-1) / 4} e^{\pi i(1-m n) / 4}\left(\frac{-n}{m}\right) e^{\pi i(1-m) / 4} \\
-670-
\end{gathered}
$$


$\mathrm{Or}$

$$
\left(\frac{-n}{m}\right)=\left(\frac{-1}{m}\right)\left(\frac{n}{m}\right)=(-1)^{(m-1) / 2}\left(\frac{n}{m}\right),
$$

d'où

$$
e^{\pi i(1-m) / 4}\left(\frac{-n}{m}\right)=e^{\pi i(m-1) / 4}\left(\frac{n}{m}\right),
$$

donc

$$
\begin{gathered}
\left(\frac{m}{n}\right)=e^{\pi i(n-1+1-m n+m-1) / 4}\left(\frac{n}{m}\right)= \\
=e^{-\pi i(n-1)(m-1) / 4}\left(\frac{n}{m}\right)=(-1)^{(n-1)(m-1) / 4}\left(\frac{n}{m}\right),
\end{gathered}
$$

cqfd.

ThÉORÈme. — Soit p un nombre premier impair. Alors

$$
\begin{gathered}
\left(\frac{2}{p}\right)=(-1)^{\left(p^{2}-1\right) / 8}= \\
1 \text { si } p \equiv \pm 1(\bmod 8) \\
-1 \text { si } p \equiv \pm 3(\bmod 8)
\end{gathered}
$$

En effet,

$$
\left(\frac{2}{p}\right)=p^{-1 / 2} e^{\pi i(p-1) / 4} g(2, p)
$$

$\mathrm{Or}$

$$
p^{-1 / 2} g(2, p)=e^{\pi i(1-2 p) / 4} 2^{-1 / 2} g(-p, 2)=e^{\pi i(1-2 p) / 4} 2^{-1 / 2}\left(1+e^{\pi i p / 2}\right)
$$

Donc

$$
\begin{gathered}
\left(\frac{2}{p}\right)=2^{-1 / 2} e^{-\pi i p / 4}\left(1+e^{\pi i p / 2}\right)=2^{-1 / 2}\left(e^{-\pi i p / 4}+e^{\pi i p / 4}\right)= \\
=\sqrt{2} \cos (\pi p / 4)
\end{gathered}
$$

Or $\cos (\pi p / 4)$ ne dépend que du reste de $p$ modulo 8 , et $\cos ( \pm \pi / 4)=\sqrt{2} / 2$ et $\cos ( \pm 3 \pi / 4)=-\sqrt{2} / 2$, d'où le résultat. 


\section{La formule sommatoire de Poisson}

3.1. Considérons l'intégrale

$$
\int_{C_{N}} \frac{e^{-\pi z^{2} t}}{e^{2 \pi i z}-1} d z=\int_{C_{N}} \phi(z, t) d z
$$

où $C_{N}$ est le rectangle avec les sommets $\pm N+\frac{1}{2} \pm i$ orienté positivement, $N$ étant un nombre entier, $N>0, t$ une variable complexe, $\Re(t)>0$.

On a

$$
\lim _{N \rightarrow \infty} \int_{C_{N}} \phi(z, t) d z=\left[\int_{-\infty-i}^{\infty-i}-\int_{-\infty+i}^{\infty+i}\right] \phi(z, t) d z=
$$

par la formule de Cauchy

$$
=\sum_{n=-\infty}^{\infty} e^{-\pi n^{2} t}=: \theta(t)
$$

3.2. D'autre part, sur la droite $z=u-i,-\infty<u<\infty$

$$
\begin{gathered}
\phi(z, t)=\frac{e^{-\pi z^{2} t}}{e^{2 \pi i z}-1}=e^{-2 \pi i z} \frac{e^{-\pi z^{2} t}}{1-e^{-2 \pi i z}}= \\
=e^{-\pi z^{2} t} \sum_{n=1}^{\infty} e^{-2 \pi i n z}=\sum_{n=1}^{\infty} e^{-\pi z^{2} t-2 \pi i n z}= \\
=\sum_{n=1}^{\infty} e^{-\pi t\left[(z+i n / t)^{2}+n^{2} / t^{2}\right]},
\end{gathered}
$$

la série convergeant uniformement. Il s'en suit

$$
\begin{gathered}
\int_{-\infty-i}^{\infty-i} \phi(z, t) d z=\sum_{n=1}^{\infty} e^{-\pi n^{2} / t} \int_{-\infty-i}^{\infty-i} e^{-\pi t(z+i n / t)^{2}} d z= \\
=\sum_{n=1}^{\infty} e^{-\pi n^{2} / t} \int_{-\infty-i}^{\infty-i} e^{-\pi t z^{2}} d z=\sum_{n=1}^{\infty} e^{-\pi n^{2} / t} \int_{-\infty}^{\infty} e^{-\pi t(u-i)^{2}} d u= \\
=\sum_{n=1}^{\infty} e^{-\pi n^{2} / t} \int_{-\infty}^{\infty} e^{-\pi t u^{2}} d u
\end{gathered}
$$

Supposons que $t$ soit réel, $t>0$. Alors (en posant $v=(\pi t)^{1 / 2} u$ )

$$
\int_{-\infty}^{\infty} e^{-\pi t u^{2}} d u=(\pi t)^{-1 / 2} \int_{-\infty}^{\infty} e^{-v^{2}} d v=t^{-1 / 2},
$$


donc ceci est vrai pout tous $t, \Re t>0$. Donc

$$
\int_{-\infty-i}^{\infty-i} \phi(z, t) d z=t^{-1 / 2} \sum_{n=1}^{\infty} e^{-\pi n^{2} / t}
$$

De même,

$$
\int_{-\infty+i}^{\infty+i} \phi(z, t) d z=-t^{-1 / 2} \sum_{n=0}^{\infty} e^{-\pi n^{2} / t}
$$

on en déduit

$$
\theta(t)=t^{-1 / 2} \sum_{n=-\infty}^{\infty} e^{-\pi n^{2} / t}=t^{-1 / 2} \theta\left(t^{-1}\right)
$$

3.3. Plus généralement, soit $f(z)$ une fonction entière telle que $|f(z)|$ décroît suffisamment rapidement quand $|\Re(z)| \rightarrow \infty$. Posons

$$
\phi(z)=\frac{f(z)}{e^{2 \pi i z}-1}
$$

Alors

$$
\left[\int_{-\infty-i}^{\infty-i}-\int_{-\infty+i}^{\infty+i}\right] \phi(z) d z=\lim _{N \rightarrow \infty} \int_{C_{N}} \phi(z) d z=\sum_{n=-\infty}^{\infty} f(n)
$$

par la formule de Cauchy.

D'un autre côté,

$$
\frac{f(z)}{e^{2 \pi i z}-1}=e^{-2 \pi i z} \frac{f(z)}{1-e^{-2 \pi i z}}=\sum_{n=1}^{\infty} f(z) e^{-2 \pi i n z}
$$

sur la droite $C_{-}=\{u-i,-\infty<u<\infty\}$, d'où

$$
\int_{-\infty-i}^{\infty-i} \phi(z) d z=\sum_{n=1}^{\infty} \int_{-\infty}^{\infty} f(u) e^{-2 \pi i n u} d u=\sum_{n=1}^{\infty} \hat{f}(-n),
$$

où l'on pose

$$
\hat{f}(t):=\int_{-\infty}^{\infty} f(u) e^{2 \pi i t u} d u
$$

De même,

$$
\int_{-\infty+i}^{\infty+i} \phi(z) d z=-\sum_{n=0}^{\infty} \hat{f}(n)
$$

Il s'en suit,

$$
\sum_{n=-\infty}^{\infty} f(n)=\sum_{n=-\infty}^{\infty} \hat{f}(n)
$$




\section{Une valeur limite d'une série thêta}

Les résultats principaux de cette Section sont 4.1.1 et 4.5. Cf. [C (a)], $[\mathrm{K}(\mathrm{a})]$.

4.1. Considérons la série

$$
\theta(t)=\sum_{n=-\infty}^{\infty} e^{-\pi n^{2} t}
$$

Ici $t$ est une variable complexe, avec $\Re(t)>0$.

Posons $t=w^{2}+\lambda i / \mu$, où $w>0, \lambda, \mu \in Z, \mu \neq 0$. Alors

$$
\theta\left(w^{2}+\lambda i / \mu\right)=\sum_{n=-\infty}^{\infty} e^{-\pi n^{2}\left(w^{2}+\lambda i / \mu\right)}=
$$

(en posant $n=k+2 \mu j$ )

$$
=\sum_{k=0}^{2 \mu-1} e^{-\lambda \pi i k^{2} / \mu} \sum_{j=-\infty}^{\infty} e^{-w^{2}(k+2 \mu j)^{2} \pi}
$$

Pour chaque $k$ on a

$$
\lim _{w \rightarrow 0} 2|\mu w| \sum_{j=-\infty}^{\infty} e^{-w^{2}(k+2 \mu j)^{2} \pi}=\int_{-\infty}^{\infty} e^{-\pi u^{2}} d u=1,
$$

donc

$$
\lim _{w \rightarrow 0} 2|\mu w| \theta\left(w^{2}+\lambda i / \mu\right)=\sum_{k=0}^{2 \mu-1} e^{-\lambda \pi i k^{2} / \mu}
$$

On désigne avec Kronecker

$$
G(\lambda i / \mu):=\frac{1}{2} \sum_{k=0}^{2 \mu-1} e^{-\lambda \pi i k^{2} / \mu},
$$

donc on arrive à :

4.1.1. THÉORÈME. -

$$
\lim _{w \rightarrow 0}|\mu w| \theta\left(w^{2}+\lambda i / \mu\right)=G(\lambda i / \mu)
$$


4.2. Maintenant prenons la valeur $t^{\prime}, t t^{\prime}=1$. On a

$$
\begin{gathered}
\left.\left(w^{2}+\lambda i / \mu\right)^{-1}=\left(w^{4}+\lambda^{2} / \mu^{2}\right)^{-1}\right)\left(w^{2}-\lambda i / \mu\right)= \\
=\left(1+w^{4} \mu^{2} / \lambda^{2}\right)^{-1}\left(w^{2} \mu^{2} / \lambda^{2}-\mu i / \lambda\right)=\left(1+w^{4} \mu^{2} / \lambda^{2}\right)^{-1}\left(w^{2} \mu^{2} / \lambda^{2}+\mu / \lambda i\right)
\end{gathered}
$$

4.3. Lemme. - Si $a, b \in R, a>0$ et $c(w)$ est une fonction réelle, $c(w)=$ $O\left(w^{4}\right)$ quand $w \rightarrow 0$, alors

$$
\lim _{w \rightarrow 0}\left[\theta\left((1+c(w))\left(a w^{2}+b i\right)\right)-\theta\left(a w^{2}+b i\right)\right]=0
$$

Preuve. On a

$$
\theta(t)=\sum_{n=-\infty}^{\infty} e^{-\pi n^{2} t}=1+2 \sum_{n=1}^{\infty} e^{-\pi n^{2} t}=1+2 \tilde{\theta}(t)
$$

Il suffit de montrer que

$$
\lim _{w \rightarrow 0}\left[\tilde{\theta}\left((1+c(w))\left(a w^{2}+b i\right)\right)-\tilde{\theta}\left(a w^{2}+b i\right)\right]=0
$$

On a

$$
(1+c(w))\left(a w^{2}+b i\right)=a w^{2}+b i+r(w),
$$

où $r(w)=O\left(w^{4}\right)$. Donc (on remarque que $1+c(w)>0$ pour $w$ assez petit)

$$
\begin{gathered}
\tilde{\theta}\left((1+c(w))\left(a w^{2}+b i\right)\right)-\tilde{\theta}\left(a w^{2}+b i\right)= \\
=\tilde{\theta}\left(a w^{2}+b i+r(w)\right)-\tilde{\theta}\left(a w^{2}+b i\right)= \\
=\sum_{n=1}^{\infty} e^{-\pi\left(a w^{2}+b i+r(w)\right) n^{2}}-\sum_{n=1}^{\infty} e^{-\pi\left(a w^{2}+b i\right) n^{2}}= \\
=\sum_{n=1}^{\infty} e^{-\pi r(w) n^{2}} e^{-\pi\left(a w^{2}+b i\right) n^{2}}-\sum_{n=1}^{\infty} e^{-\pi\left(a w^{2}+b i\right) n^{2}}= \\
=\sum_{n=1}^{N}\left(e^{-\pi r(w) n^{2}}-1\right) e^{-\pi\left(a w^{2}+b i\right) n^{2}}+\sum_{n=N+1}^{\infty} e^{-\pi\left(a w^{2}+b i+r(w)\right) n^{2}}- \\
-\sum_{n=N+1}^{\infty} e^{-\pi\left(a w^{2}+b i\right) n^{2}}
\end{gathered}
$$

(a) La première somme. Pour $n \leqslant N,\left|r(w) n^{2}\right| \leqslant\left|r(w) N^{2}\right|$. On a pour chaque $a$ réel

$$
\left|e^{-a}-1\right|=\left|-a+\frac{a^{2}}{2}-\ldots\right| \leqslant|a|+\frac{|a|^{2}}{2}+\ldots=e^{|a|}-1,
$$


donc

$$
\left|e^{-\pi r(w) n^{2}}-1\right| \leqslant e^{\left|\pi r(w) n^{2}\right|}-1 \leqslant e^{\left|\pi r(w) N^{2}\right|}-1(n \leqslant N)
$$

Par hypothèse il existe une constante $\tilde{C}>0$ telle que $\left|\pi r(w) N^{2}\right| \leqslant \tilde{C} w^{4} N^{2}$, donc il existe $C>0$ tel que

$$
\left|e^{\left|\pi r(w) N^{2}\right|}-1\right|<C w^{4} N^{2}
$$

D'un autre côté,

$$
\left|e^{-\pi\left(a w^{2}+b i\right) n^{2}}\right|=e^{-\pi a w^{2} n^{2}}<1
$$

Donc

$$
\left|\sum_{n=1}^{N}\left(e^{-\pi r(w) n^{2}}-1\right) e^{-\pi\left(a w^{2}+b i\right) n^{2}}\right| \leqslant C w^{4} N^{3}
$$

Posons $w=N^{-\alpha}(\alpha>0)$; alors on obtient

$$
\left|\sum_{n=1}^{N}\left(e^{-\pi r\left(N^{-\alpha}\right) n^{2}}-1\right) e^{-\pi\left(a N^{-2 \alpha}+b i\right) n^{2}}\right| \leqslant C N^{-4 \alpha+3}
$$

Ceci tend vers 0 si $4 \alpha-3>0$, i.e. si $\alpha>3 / 4$.

(b) Maintenant considérons la troisième somme dans (4.3.1). On a

$$
\begin{gathered}
\left|\sum_{n=N+1}^{\infty} e^{-\pi\left(a N^{-2 \alpha}+b i\right) n^{2}}\right|<\sum_{k=0}^{\infty} e^{-a \pi N^{-2 \alpha}(N+k)^{2}}< \\
\quad<\sum_{k=0}^{\infty} e^{-a \pi\left(N^{2-2 \alpha}+2 k N^{1-2 \alpha}\right)}=\frac{e^{-a \pi N^{2-2 \alpha}}}{1-e^{-2 a \pi N^{1-2 \alpha}}}
\end{gathered}
$$

Or,

$$
\frac{1}{1-e^{-2 a \pi N^{1-2 \alpha}}}=O\left(N^{2 \alpha-1}\right)(\alpha>1 / 2)
$$

Donc (4.3.2) tend vers 0 si $1 / 2<\alpha<1$.

De même, on établit que la deuxième somme tend vers 0 sous la même hypothèse $1 / 2<\alpha<1$.

Le lemme en découle. 
4.4. Revenons à 4.2. En utilisant le lemme,

$$
\begin{gathered}
\lim _{w \rightarrow 0}|\mu w| \theta\left(\left(w^{2}+\lambda i / \mu\right)^{-1}\right)= \\
=\lim _{w \rightarrow 0}|\lambda w \mu / \lambda| \theta\left(w^{2} \mu^{2} / \lambda^{2}+\mu / \lambda i\right)=G(\mu / \lambda i)
\end{gathered}
$$

La formule de Poisson nous dit

$$
\theta(t)=t^{-1 / 2} \theta\left(t^{-1}\right)
$$

Donc

$$
\begin{gathered}
G(\lambda i / \mu)=\lim _{w \rightarrow 0}|\mu w| \theta\left(w^{2}+\lambda i / \mu\right)= \\
=\lim _{w \rightarrow 0}|\mu w|\left(w^{2}+\lambda i / \mu\right)^{-1 / 2} \theta\left(\left(w^{2}+\lambda i / \mu\right)^{-1}\right)=(\lambda i / \mu)^{-1 / 2} G(\mu / \lambda i)
\end{gathered}
$$

Autrement dit,

$$
(\lambda i / \mu)^{1 / 2} G(\lambda i / \mu)=G(\mu / \lambda i)
$$

On a évidemment ici un abus de notation car $G(\lambda i / \mu)$ depend de $\lambda, \nu$. Pour l'éviter, on peut ${ }^{2}$ poser, pour $r=\lambda / \mu$,

$$
\tilde{G}(i r)=\frac{1}{2 \mu} \sum_{j=0}^{2 \mu-1} e^{-\pi i r / j^{2}}
$$

Alors (4.4.1) se reécrit en

4.5. ThÉORÈME. - On $a$ :

$$
(i / r)^{1 / 2} \tilde{G}(i / r)=\tilde{G}(-i r)
$$

C'est une forme de (1.5.4).

\section{La formule de produit de Gauss}

5.1. Le but de cette Section est la détermination du signe de la somme de Gauss par la méthode de Gauss, cf. [G]. On pose

$$
(m, \mu)=\frac{\left(1-x^{m}\right)\left(1-x^{m-1}\right) \cdot \ldots \cdot\left(1-x^{m-\mu+1}\right)}{(1-x)\left(1-x^{2}\right) \cdot \ldots\left(1-x^{\mu}\right)} \in C\left[x, x^{-1}\right]
$$

("les coefficients $x$-binomiaux"). Ici $\mu \in N, m \in Z$.

(2) en suivant une suggestion du rapporteur 
Exemples : $(m, 0)=1$;

$$
(-1, \mu)=\prod_{i=1}^{\mu} \frac{1-x^{-i}}{1-x^{i}}=(-1)^{\mu} x^{-\mu(\mu+1) / 2} \in C\left[x^{-1}\right]
$$

En général, $(-m, \mu) \in C\left[x^{-1}\right]$, et à la limite

$$
(-\infty, \mu):=\lim _{m \rightarrow \infty}(-m, \mu)=\frac{1}{(1-x)\left(1-x^{2}\right) \cdot \ldots \cdot\left(1-x^{\mu}\right)} \in C\left[\left[x^{-1}\right]\right]
$$

Si $m \in N,(m, \mu)=0$ si $\mu>m$, et

$$
(m, \mu)=(m, m-\mu)
$$

5.2. On a

$$
(m, \mu)=(m-1, \mu)+x^{m-\mu}(m-1, \mu-1)
$$

Il s'en suit que si $m \in N, m>\mu+1$, alors

$$
(m, \mu+1)=\sum_{i=0}^{m-\mu-1}(\mu+i, \mu) x^{i}
$$

On en déduit par récurrence sur $\mu$ que $(m, \mu)$ est un polynôme en $x$ si $m \in N$.

5.3. On pose

$$
f(x, m)=1-\frac{1-x^{m}}{1-x}+\frac{\left(1-x^{m}\right)\left(1-x^{m-1}\right)}{(1-x)\left(1-x^{2}\right)}-\ldots=\sum_{\mu=0}^{\infty}(-1)^{\mu}(m, \mu) \in C\left(\left(x^{-1}\right)\right)
$$

Si $m \in N$, la somme est finie :

$$
f(x, m)=\sum_{\mu=0}^{m}(-1)^{\mu}(m, \mu) \in C[x] \subset C\left(\left(x^{-1}\right)\right)
$$

On a $f(x, 0)=1, f(x, 1)=0$.

5.4. Il découle de (5.2.1) :

$$
\begin{gathered}
(m, 0)=1 \\
-(m, 1)=-(m-1,1)-x^{m-1} \\
(m, 2)=(m-1,2)+x^{m-2}(m-1,1), \text { etc. } \\
-678-
\end{gathered}
$$


d'où

$$
f(x, m)=\sum_{i=0}^{\infty}(-1)^{i}\left(1-x^{m-1-i}\right)(m-1, i)
$$

Par contre,

$$
\left(1-x^{m-1-i}\right)(m-1, i)=\left(1-x^{m-1}\right)(m-2, i),
$$

d'où

$$
f(x, m)=\left(1-x^{m-1}\right) f(x, m-2)
$$

5.5. Supposons que $m \in N$. Alors si $m$ est pair (5.4.1) implique que

$$
f(x, m)=(1-x)\left(1-x^{3}\right) \cdot \ldots \cdot\left(1-x^{m-1}\right)=\prod_{j=0}^{(m-2) / 2}\left(1-x^{2 j+1}\right)
$$

Par contre, si $m$ est impair, $f(x, m)=0$ car $f(x, 1)=0$.

5.6. Si $m=-2 k, k \in Z, k>0$, on obtient

$$
f(x,-2 k)=\frac{1}{\left(1-x^{-1}\right)\left(1-x^{-3}\right) \cdot \ldots \cdot\left(1-x^{-2 k+1}\right)} \in C\left[\left[x^{-1}\right]\right]
$$

En passant à la limite pour $k \rightarrow \infty$ dans la topologie $\left(x^{-1}\right)$-adique, on aura

$$
\lim _{k \rightarrow \infty} f(x,-2 k)=\sum_{i=0}^{\infty} \frac{1}{(x-1)\left(x^{2}-1\right) \cdot \ldots \cdot\left(x^{i}-1\right)}=\frac{1}{\prod_{n=0}^{\infty}\left(1-x^{-2 n-1}\right)}
$$

De même,

$$
f(x,-2 k-1)=\frac{f(x,-1)}{\left(1-x^{-2}\right)\left(1-x^{-4}\right) \ldots\left(1-x^{-2 k}\right)}
$$

où

$$
f(x,-1)=1+x^{-1}+x^{-3}+x^{-6}+\ldots=\sum_{n=0}^{\infty} x^{-n(n+1) / 2},
$$

d'où

$$
\lim _{k \rightarrow \infty} f(x,-2 k-1)=\frac{f(x,-1)}{\prod_{k=1}^{\infty}\left(1-x^{-2 k}\right)}
$$

Or, les deux limites coïncident :

$$
\lim _{k \rightarrow \infty} f(x,-2 k)=\lim _{k \rightarrow \infty} f(x,-2 k-1)=\lim _{n \rightarrow \infty} f(x,-n)=: f(x,-\infty),
$$


d'où

$$
f(x,-1)=1+x^{-1}+x^{-3}+x^{-6}+\ldots=\frac{\left(1-x^{-2}\right)\left(1-x^{-4}\right) \cdot \ldots}{\left(1-x^{-1}\right)\left(1-x^{-3}\right) \cdot \ldots},
$$

ou bien

$$
\sum_{n=0}^{\infty} x^{n(n+1) / 2}=1+x+x^{3}+x^{6}+\ldots=\frac{\left(1-x^{2}\right)\left(1-x^{4}\right) \cdot \ldots}{(1-x)\left(1-x^{3}\right) \cdot \ldots} \in C[[x]],
$$

les deux cotés convergent pour $|x|<1$.

On peut récrire

$$
\sum_{n=0}^{\infty} x^{n(n+1) / 2}=\frac{1}{2} \sum_{n=-\infty}^{\infty} x^{n(n+1) / 2}
$$

et

$$
\begin{gathered}
\frac{\prod_{i=1}^{\infty}\left(1-x^{2 i}\right)}{\prod_{i=1}^{\infty}\left(1-x^{2 i-1}\right)}=\frac{\prod_{i=1}^{\infty}\left(1-x^{2 i}\right)^{2}}{\prod_{i=1}^{\infty}\left(1-x^{i}\right)}= \\
=\frac{\prod_{i=1}^{\infty}\left(1+x^{i}\right)^{2}\left(1-x^{i}\right)^{2}}{\prod_{i=1}^{\infty}\left(1-x^{i}\right)}=\prod_{i=1}^{\infty}\left(1+x^{i}\right)^{2}\left(1-x^{i}\right),
\end{gathered}
$$

donc

$$
\frac{1}{2} \sum_{n=-\infty}^{\infty} x^{n(n+1) / 2}=\prod_{i=1}^{\infty}\left(1+x^{i}\right)^{2}\left(1-x^{i}\right)
$$

ce qui est une formule standard de la théorie des fonctions thêta, cf. Jacobi, Fund., no. 66, (4) ; [W], p. I, ch. IV, §9, (28).

Plus généralement, pour tout $m \in Z$, on obtient $f(x, m)=f(x,-\infty)\left(1-x^{m-1}\right)\left(1-x^{m-3}\right) \cdot \ldots=\frac{\left(1-x^{m-1}\right)\left(1-x^{m-3}\right) \cdot \ldots}{\left(1-x^{-1}\right)\left(1-x^{-3}\right) \cdot \ldots}$

5.7. Maintenant soit $n$ un entier positif impair ; posons $m=n-1$, et soit $r$ une racine primitive de l'équation $x^{n}=1$; posons $x=r$. On a

$$
(n-1, \mu)=\frac{\left(1-r^{n-1}\right)\left(1-r^{n-2}\right) \cdot \ldots \cdot\left(1-r^{n-\mu}\right)}{(1-r)\left(1-r^{2}\right) \cdot \ldots \cdot\left(1-r^{\mu}\right)}
$$

Or :

$$
\begin{aligned}
\frac{1-r^{n-i}}{1-r^{i}} & =\frac{1-r^{-i}}{1-r^{i}}=-r^{-i} \\
& -680-
\end{aligned}
$$


d'où

$$
(n-1, \mu)=(-1)^{\mu} r^{-\mu(\mu+1) / 2}
$$

Donc

$f(r, n-1)=1+r^{-1}+r^{-3}+r^{-6}+\ldots r^{-n(n-1) / 2}=(1-r)\left(1-r^{3}\right) \ldots \cdot\left(1-r^{n-2}\right)$,

$\operatorname{par}(5.5 .1)$.

5.8. On peut remplacer dans (5.7.1) $r$ par n'importe quel $r^{\lambda}$ où $(\lambda, n)=$ 1 ; par exemple par $r^{-2}$ :

$$
\begin{aligned}
& \sum_{i=0}^{n-1} r^{i(i+1)}=1+r^{2}+r^{6}+r^{12}+\ldots+r^{n(n-1)} \\
& =\left(1-r^{-2 \cdot 1}\right)\left(1-r^{-2 \cdot 3}\right) \cdot \ldots \cdot\left(1-r^{-2(n-2)}\right)
\end{aligned}
$$

Soit $n=2 k+1$; on a

$$
1+3+5+\ldots+(2 k-1)=k^{2},
$$

i.e.

$$
1+3+\ldots+(n-2)=\frac{(n-1)^{2}}{4}
$$

Multiplions les deux côtés de (5.8.1) par

$$
1 \cdot r \cdot r^{3} \cdot \ldots \cdot r^{n-2}=r^{(n-1)^{2} / 4}
$$

Remarquons que

$$
i(i+1)+\frac{(n-1)^{2}}{4} \equiv \frac{(n-2 i-1)^{2}}{4}(\bmod n),
$$

donc à gauche on obtient

$$
r^{k^{2}}+r^{(k-1)^{2}}+\ldots+r+1+r+r^{4}+\ldots+r^{k^{2}}=\sum_{i=0}^{n-1} r^{i^{2}}
$$

car $i^{2} \equiv(n-i)^{2}(\bmod n)$. Il s'en suit que

$1+r+r^{4}+\ldots+r^{(n-1)^{2}}=\left(r-r^{-1}\right)\left(r^{3}-r^{-3}\right)\left(r^{5}-r^{-5}\right) \cdot \ldots \cdot\left(r^{n-2}-r^{-n+2}\right)$

5.9. Soit $p$ un nombre premier impair, $p=2 k+1, \zeta=e^{2 \pi i / p}$. Considérons la somme de Gauss

$$
\begin{gathered}
g(\zeta)=\sum_{a=1}^{p-1}\left(\frac{a}{p}\right) \zeta^{a}=\sum_{\rho \in R} \zeta^{\rho}-\sum_{\nu \in N} \zeta^{\nu}, \\
-681-
\end{gathered}
$$


où $R$ (resp. $N$ ) est l'ensemble des résidus (resp. des non-résidus) quadratiques. Puisque

$$
1+\sum_{\rho \in R} \zeta^{\rho}+\sum_{\nu \in N} \zeta^{\nu}=\sum_{a=0}^{p-1} \zeta^{a}=0
$$

on a

$$
g(\zeta)=1+2 \sum_{\rho \in R} \zeta^{\rho}=\sum_{n=0}^{p-1} \zeta^{a^{2}}
$$

Donc

$$
g(\zeta)=\prod_{s \in S}\left(\zeta^{s}-\zeta^{-s}\right)=(2 i)^{k} \prod_{s \in S} \sin 2 \pi s / p
$$

où

$$
S=\{1,3,5, \ldots, 2 k-1\}, \quad \operatorname{Card}(S)=k=(p-1) / 2
$$

Supposons que $k$ est impair, $k=2 j+1$, i.e. $p=4 j+3$. On a

$$
S=\{1,3,5, \ldots, 2 j+1\} \coprod\{k+2, k+4, \ldots, k+2 j\},
$$

où $k+2=p-k+1 \equiv-(k-1)(\bmod p)$, etc., d'où

$$
\prod_{s \in S} \sin 2 \pi s / p=(-1)^{j} \prod_{a=1}^{k} \sin 2 \pi a / p,
$$

donc

$$
g(\zeta)=(2 i)^{2 j+1}(-1)^{j} \prod_{a=1}^{k} \sin 2 \pi a / p=i 2^{(p-1) / 2} \prod_{a=1}^{(p-1) / 2} \sin 2 \pi a / p
$$

De même, si $k=2 j$, i.e. $p=4 j+1$,

$$
g(\zeta)=2^{(p-1) / 2} \prod_{a=1}^{(p-1) / 2} \sin 2 \pi a / p
$$

Dans le produit $\prod_{a=1}^{(p-1) / 2} \sin 2 \pi a / p$, on a $0<a<p / 2$, donc $0<$ $2 \pi a / p<\pi$, d'où

$$
\prod_{a=1}^{(p-1) / 2} \sin 2 \pi a / p>0
$$

D'autre part il est bien connu que $|g(\zeta)|^{2}=p$. Il s'en suit : 
5.10. THÉORÈME. -

$$
g(\zeta)=\sqrt{p} \quad \text { si } p \equiv 1(\bmod 4)
$$

et

$$
g(\zeta)=i \sqrt{p} \quad \text { si } p \equiv 3(\bmod 4)
$$

\section{Bibliographie}

[C] CAuchy (A.). - (a) Méthode simple et nouvelle pour la détermination complète des sommes alternées, formées avec les racines primitives des équations binômes, C.R., t. X, p. 560 (6 avril 1840) - Euvres complètes d'Augustin Cauchy, I-ière série, t. V, Paris, Gauthier-Villars, MDCCCLXXXV, pp. 152-166. (b) Sur la sommation de certaines puissances d'une racine primitive d'une équation binôme, et en particulier, des puissances qui offrent pour exposants les résidus cubiques inférieurs au module donné, C.R. t. X, p. 594 (13 avril 1840) - Euvres, ibid., pp. 166-180. (c) Sur une loi de réciprocité qui existe entre certaines fonctions, Bull. de la Société Philomathique, pp. 121-124, 1817 - Euvres, II-ième sér., t. II, pp. 223-227 (d) Seconde note sur les fonctions réciproques, Bull. Soc. Phil., p. 188-191, 1818 - Euvres, ibid., p. 228-232.

[Ch] Chandrasekharan (K.). - Introduction to analytic number theory, Springer, Die Grundlehren der mathematischen Wissenschaften, Band 148, (1968).

[D] G. Lejeune Dirichlet. - Sur l'usage des intégrales définies dans la sommation des séries finies ou infinies, J. Crelle, Band XVII (1837), 1. Heft, ss. p. 57-67.

[G] Gauss (K.-F.). - Summatio quarumdam serierum singularium, Commentationes societatis regiae scientarium Gottingensis recentiores, Vol. 1, Gottingae MDCCCXI — Werke, Bd. II, p. 9-45.

[H] Hecke (E.). - Vorlesungen über die Theorie der algebraischen Zahlen, Première edition : Leipzig, 1923 ; deuxième edition : New York, 1948, Chelsea, Bronx, (1970).

[K] Kronecker (L.). - (a) Über den vierten Gauss'schen Beweis des Reciprotätsgesetzes für die quadratischen Reste, Monatsberichte der Königlich Preussischen Academie der Wissenschaften zu Berlin vom Jahre 1880, ss. 686-698 and ss. 854-860 - Werke, Chelsea, NY, 1968, Bd. IV, pp. 277-294. (b) Summirung der Gauss'schen Reihen $\sum_{h=0}^{h=n-1} e^{2 h^{2} \pi i / n}$, Crelle Journal für reine und angewandte Mathematik, 105, ss. 267-268 — Werke, Bd. IV, pp. 297-300. (c) Sur une formule de Gauss, Liouville Journal de mathématiques pures et appliquées, Ser. II, tome 1, pp. 392-395 = Werke Bd. IV, p. 173-175. (d) Über die Dirichletsche methode der Wertbestimmung der Gauss'schen Reihen, Festschrift der Mathematischen Gesellschaft in Hamburg aus dem Jahre 1890, ss. 32-36 — Werke, Bd. IV, p. 303-307.

[S] Siegel (C.-L.). - Über Riemanns Nachlaß zur analytischen Zahlentheorie, Quellen und Studien zur Geschichte der Mathematik, Astronomie und Physik, 2 (1932), p. 45-80 - Gesammelte Abhandlungen, t. I, 266.

[W] WeIL (A.). - Elliptic functions according to Eisenstein and Kronecker, Springer (1976). 